\title{
Полевой транзистор на протонной проводимости пленок оксида графена и нафиона
}

\author{
(С) В.А. Смирнов ${ }^{1}$, А.Д. Мокрушин ${ }^{2}$, Н.Н. Денисов ${ }^{1}$, Ю.А. Добровольский ${ }^{1}$ \\ ${ }^{1}$ Иститут проблем химической физики Российской академии наук, \\ 142432 Черноголовка, Московская обл., Россия \\ ${ }^{2}$ Институт проблем технологии микроэлектроники и особочистых материалов Российской академии наук, \\ 142432 Черноголовка, Московская обл., Россия \\ E-mail: vas@icp.ac.ru
}

(Получена 28 марта 2017 г. Принята к печати 17 апреля 2017 г.)

В модели полевого транзистора исследована протонная проводимость в пленках оксида графена и нафиона в зависимости от влажности и напряжений на электродах. Электрические характеристики пленок подобны друг другу, но подвижность положительных зарядов в нафионе и усиление тока на 2-3 порядка выше, чем в оксиде графена. В пленках оксида графена отрицательно-ионный ток при положительном напряжении смещения составляет заметную величину от протонного (до $\sim 10 \%$ ), в то время как в пленках нафиона он практически отсутствует $(<1 \%)$.

DOI: $10.21883 /$ FTP.2018.03.45624.8594a

\section{1. Введение}

Оксид графена (ОГ) является основным исходным материалом для получения графеноподобных материалов со свойствами графена [1]. Однако и сам ОГ обладает достаточно интересными свойствами, которые делают возможным его практическое использование [2-8]. В работе [9] для широкого спектра приложений предложено оксид графена наносить на твердые и гибкие подложки путем двумерной печати. В работе [10] было предложено использовать восстановленный ОГ в качестве электрода суперконденсатора, а в работе [11] описан первый образец суперконденсатора, в котором ОГ использован как сепаратор. В работах $[12,13]$ исследовалась проводимость пленок ОГ в зависимости от влажности и типа растворителя, в парах которого проводились измерения, и было установлено, что проводимость ОГ имеет протонный характер, в то время как в сухой атмосфере ОГ является изолятором. Протонный характер проводимости подтверждается как изотопным эффектом при измерении проводимости в парах $\mathrm{H}_{2} \mathrm{O}$ и $\mathrm{D}_{2} \mathrm{O}[11,13]$, так и сравнением проводимости пленок ОГ с проводимостью типичного протонного проводника. Проводимость пленок ОГ во влажной атмосфере исследовалась также в работе [14], в которой наблюдалась экспоненциальная зависимость проводимости от влажности.

Полевой эффект в транзисторе на основе графена впервые был изучен в основополагающей работе Новоселова, Гейма и сотр. [15], в которой был реализован и детально исследован полевой транзистор с малой толщиной двумерного углеродного канала вплоть до графенового монослоя, высаженного на окисленную поверхность монокристаллической пластинки кремния. В работе были исследованы также магнитные явления в таких транзисторах. Эта работа вызвала взрывной интерес к объектам типа графена, а результаты исследований можно найти в обзорных статьях [16-18]. Исследования полевого транзистора на основе графена и окисленного кремния проведены в работах [19-21]. В работе [19] исследована подвижность носителей заряда в широком диапазоне температур. В работе [20] наблюдали смещение дираковской точки при обработке поверхности графена пучком электронов с энергией 30 кэВ. В работе [21] в полевых транзисторах использовали модифицированный графен (гетероструктуры), что позволило напряжением на затворе изменять величину рабочего тока на 4-5 порядков.

В литературе ОГ в полевом транзисторе обычно использовался в качестве изолирующего слоя [22-24]. В работе [23] на слое ОГ водородной плазмой создавался проводящий графеновый слой, а ОГ служил изолирующим слоем на затворе. ОГ использовался также в качестве изолирующего слоя в транзисторе, нанесенном на гибкий пластик, в котором в качестве активного слоя и электродов использовался графен [24]. Хорошие характеристики наблюдаются в полевом транзисторе на основе композита ОГ с поливинилкарбазолом [25]. В некоторых работах в многослойных полевых транзисторах вводили дополнительный слой ОГ, который создавал эффект памяти [26-28].

В работах $[29,30]$ был изучен эффект поперечного электрического поля на протонную и электронную проводимости пленок ОГ в различных исходных состояниях: при наличии лишь протонной составляющей тока во влажной атмосфере и при частичном восстановлении ОГ до появления электронной составляющей тока в сухом образце. В литературе влияние поперечного электрического поля на протонную проводимость исследовалось для некоторых протонных проводников: полисахариды [31] и нафион [32,33]. Протонный проводник нафион широко используется в топливных элементах в качестве мембран, которые обладают высокой протонной проводимостью и возможностью регулирования их набухания в воде [34,35]. В данной работе поставлена задача исследовать эффект поперечного электрического поля на протонную составляющую тока в пленках ОГ и 

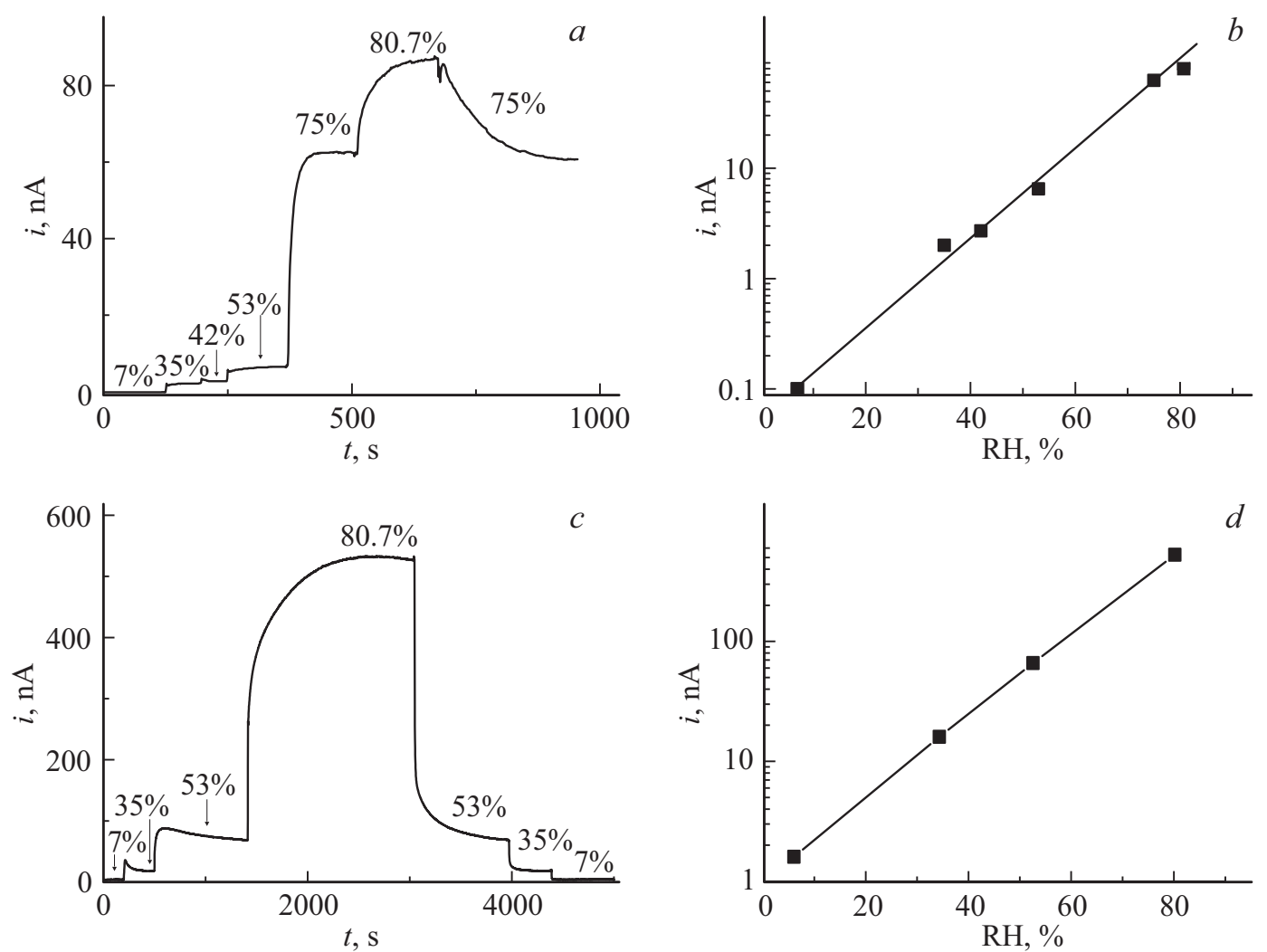

Рис. 1. Поведение тока в пленках ОГ $(a, b)$ и нафиона $(c, d)$ в зависимости от влажности среды. $a$, $c$ - изменение тока при ступенчатом во времени $t$ изменении влажности (значения RН указаны); $b, d-$ зависимость стационарного тока от влажности. Напряжение $U=0.1 \mathrm{~B}$.

сравнить его с аналогичным эффектом в пленках нафиона - типичного представителя полимерных протонных проводников.

\section{2. Методика эксперимента}

Получение ОГ и используемые экспериментальные приемы приведены в [12,13]. Для приготовления пленок нафиона использовался коммерческий раствор: D1021 Nafion Dispersion - Water based $1100 \mathrm{EW}$ at $10 \mathrm{wt} \%$. Электрические свойства пленок исследовались двухэлектродным методом, что согласуется с измерениями в схеме полевого транзистора. Пленки ОГ и нафиона были сформированы на ячейке с золотыми электродами путем нанесения соответствующей водной суспензии и высушивания при комнатных условиях. Толщина пленок ( 1 мкм) задавалась концентрацией исходного раствора. Электрические измерения проводились на потенциостате P-20X Elins.

\section{3. Экспериментальные результаты и их обсуждение}

\section{1. Протонная проводимость}

В пленках ОГ, нанесенных на электроды и высушенных при комнатной температуре, в парах воды можно наблюдать электропроводность, и в этих условиях пленки ОГ являются типичными протонными проводниками [11-14]. На рис. 1, a показано поведение тока $i$ в пленке ОГ при помещении ее в условия с различной относительной влажностью (relative humidity, RH), а на рис. $1, b$ приведена зависимость тока от влажности, и эта зависимость спрямляется в логарифмических координатах. Аналогичные токовые зависимости наблюдаются и для пленок нафиона (рис. $1, c, d)$ - зависимость тока от влажности также спрямляется в логарифмических координатах. Эти результаты согласуются с результатами работ [36-38] по измерению зависимости протонной проводимости мембран нафиона от влажности, однако в цитируемых работах не наблюдалось строгой линейной зависимости логарифма протонной проводимости от влажности.

Следует отметить, что при высокой влажности и большом напряжении на электродах могут возникать нелинейные эффекты - неконтролируемое увеличение тока вплоть до электрического пробоя.

\section{2. Температурные характеристики проводимости}

На рис. 2 представлены температурные зависимости протонной проводимости пленок ОГ (кривая 1) и нафиона (кривая 2). В диапазоне температур $T=7-36^{\circ} \mathrm{C}$ 


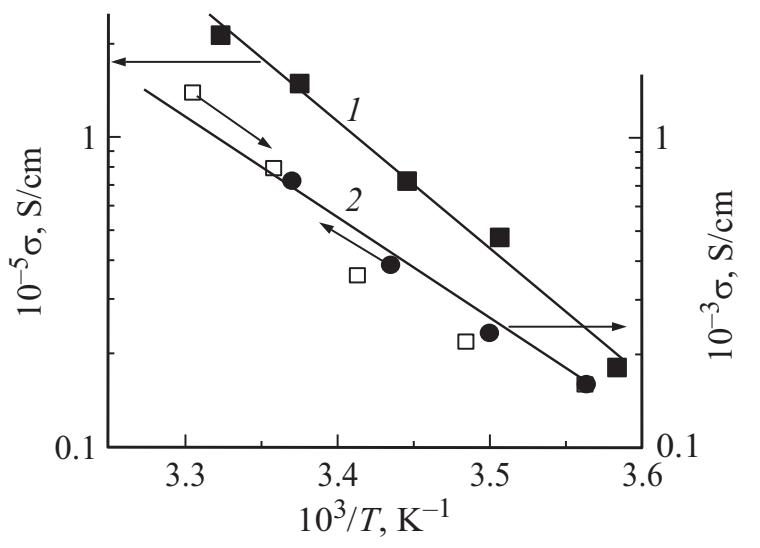

Рис. 2. Температурные зависимости протонной проводимости для пленок ОГ (1) и нафиона (2). 1: влажность $75 \%, U=0.3 \mathrm{~B}$. 2: влажность $35 \%, U=0.1 \mathrm{~B}$, приведены данные для режимов охлаждения и нагревания.

эти зависимости $\sigma(T)$ подчиняются закону Аррениуса с энергией активации $E_{a}=(0.91 \pm 0.05)$ эВ для ОГ и $E_{a}=(0.89 \pm 0.05)$ эВ для нафиона. Эти значения близки друг к другу, а также к литературным данным по графеновым материалам.

Близкая по величине энергия активации протонной проводимости $E_{a}=0.83$ эВ наблюдалась в работе [39] для мембран, изготовленных из ОГ-бумаги. Такое же близкое значение энергии активации для протонного транспорта через графеновый монослой наблюдали в работе [40]: $E_{a}=(0.78 \pm 0.03)$ эВ.

Энергии активации для протонной проводимости мембран нафиона могут существенно различаться в зависимости от способа предварительной обработки мембран. Для мембран нафиона, предварительно обработанных в кипящей воде с различными добавками, энергия активации протонной проводимости составляет 10.7 кДж/моль $(0.11$ эB) [41]. В работе [42] энергия активации миграции протонов при RH 100\% для мембран нафиона, обработанных в различных условиях, составляла величину всего $1.1-1.8$ кДж/моль $(0.01-0.018$ эВ). В нашем случае предварительной термической обработки пленки нафиона не проводилось: пленки нафиона и ОГ сушились в одинаковых условиях при комнатной температуре, а перед измерениями помещались на 30 мин в сухую атмосферу, с относительной влажностью $<7 \%$.

\section{3. Релаксационные процессы}

Ранее в работе [30] были изучены как электронная, так и протонная проводимость пленок ОГ. Различие свойств электронной и протонной проводимости сказывается и в релаксационных процессах. На рис. 3, $a$ приведены переходные токовые характеристики при ступенчатом во
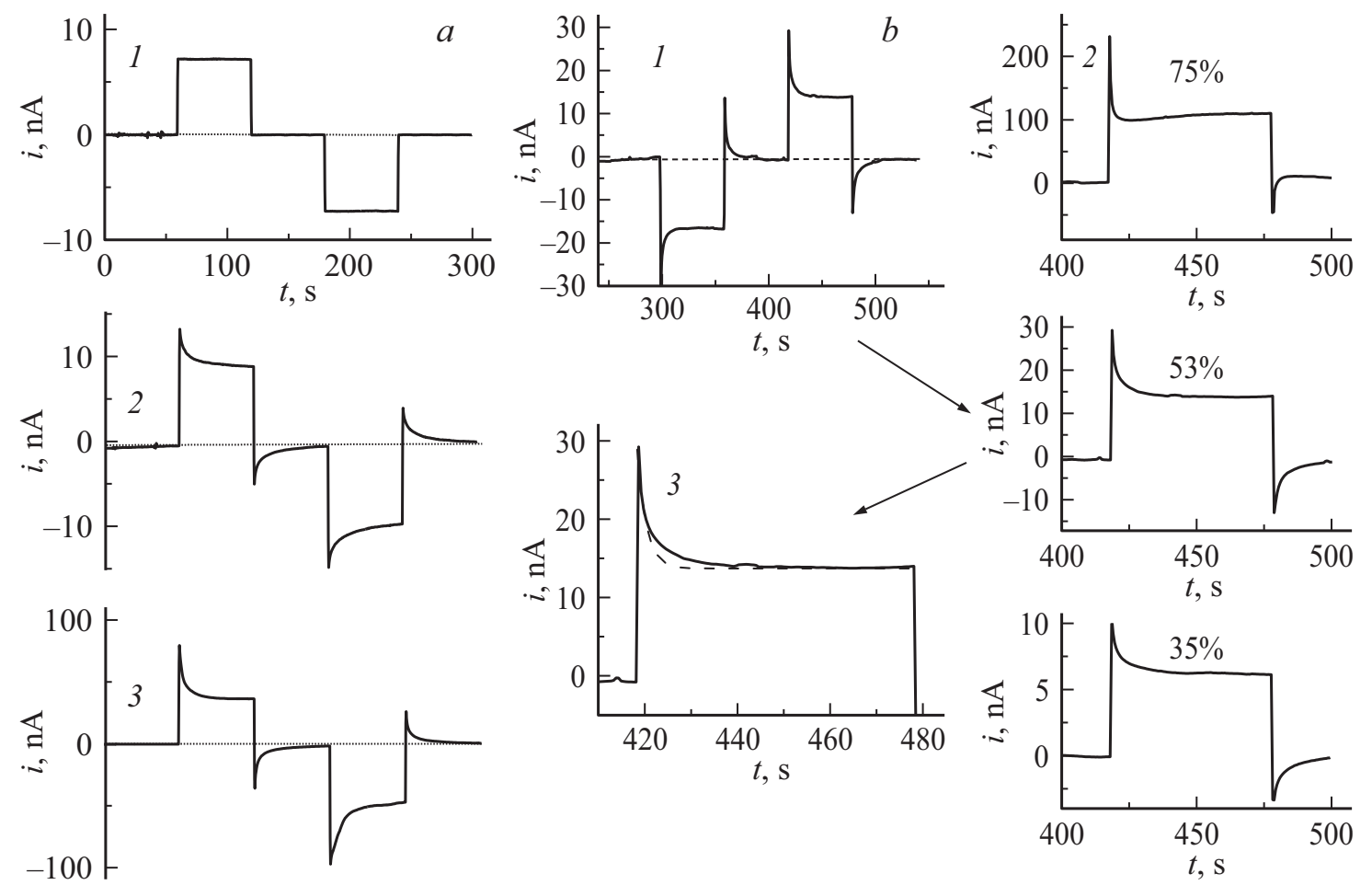

Рис. 3. $a-$ переходные характеристики тока в пленке ОГ при ступенчатом изменении во времени $t$ напряжения на +1 и $-1 \mathrm{~B}$, интервал 60 с: 1 - электронная проводимость пленки при RH 7\%; 2 - суммарная проводимость при RH 53\%; 3 - суммарная проводимость при RH 75\%. $b$ - зависимости тока в пленке нафиона при ступенчатом изменении напряжения на электродах $0 \rightarrow(-100 \mathrm{MB}) \rightarrow 0 \rightarrow(100 \mathrm{MB}) \rightarrow 0$ при различных влажностях (значения RH указаны). 
времени $t$ изменении напряжения на образце. Кривая 1 соответствует изменению электронного тока (RH 7\%) в частично восстановленной пленке ОГ при подаче на электроды напряжения +1 и -1 В с минутным интервалом. Видно, что кривые изменения тока прямоугольные, что характерно для безынерционной составляющей тока. При помещении того же образца во влажную атмосферу c RH 53\% (кривая 2) в образце появляется протонная составляющая, сравнимая по величине с электронной проводимостью. В переходных характеристиках появляется релаксационная составляющая протонного тока. Дальнейшее увеличение влажности до RH 75\% (кривая 3) значительно увеличивает протонный ток, и его релаксационная составляющая становится сравнимой по величине со стационарным электронным током. Релаксационная составляющая тока при протонной проводимости, по-видимому, обусловлена как дипольной поляризацией молекул воды, так и поляризацией отдельных фрагментов нанолиста ОГ. Релаксация не описывается моноэкспоненциальной зависимостью, а время релаксации (на полувысоте) составляет несколько секунд. За такие процессы могут быть ответственны заряженные фрагменты с присоединенной водой, а релаксация обусловлена не только электронной составляющей, но и структурной, т.е. их поворотом и частичным перемещением. Такими фрагментами могут быть, например, группы $\mathrm{H}_{2} \mathrm{O}_{2}^{-}$, возникающие в процессе присоединения молекулы воды к гидроксильной группе, а протон от этой группы участвует в проводимости.

При помещении измерительной ячейки с сухой пленкой нафиона во влажную атмосферу вначале возникает поляризационный пик проводимости, обусловленный в основном электронной поляризацией и дипольной поляризацией молекул воды, попавшей в пленку. На рис. 3, $b$ представлены переходные токовые характеристики при ступенчатом изменении напряжения в пленке нафиона, находящейся во влажной атмосфере. Как следует из рис. $3, b$, ступенчатое изменение напряжения вызывает скачок тока, который в первый момент обусловлен электронной составляющей с временем спада $\tau \ll 1$ с. Дальнейший спад тока обусловлен дипольной поляризацией, а его существенное отличие от экспоненциального вида (рис. $3, b$, штриховая кривая) означает, что в этом процессе участвуют различные диполи - это могут быть как молекулы воды, так и полярные полимерные фрагменты. Действительно, переходные процессы отличаются друг от друга при нахождении пленки в среде с различной влажностью (рис. 3,b) - при увеличении влажности увеличивается вклад быстрой электронной поляризации, что говорит о наличии свободной воды. В работе [43] показано, что переходные поляризационные характеристики в мембранах нафиона при относительной влажности 76\% в атмосфере водорода можно описать идеальной поляризацией электродов, которая эквивалентна разряду конденсатора через омическое сопротивление - за этот процесс ответственна электронная поляризация.

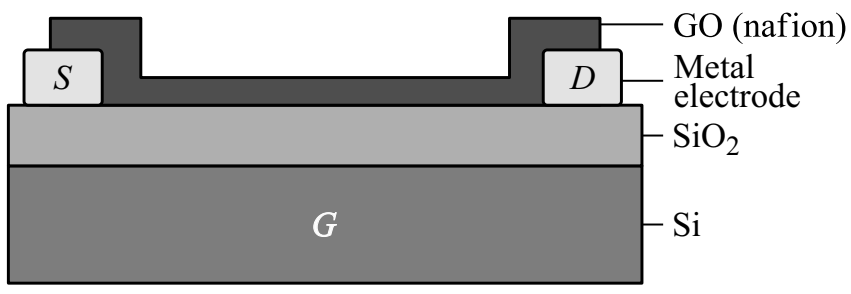

Рис. 4. Схема полевого транзистора. $S-$ исток, $D-$ сток, $G$ - затвор.

\section{4. Модель полевого транзистора}

Для исследования влияния поперечного электрического поля на протонную проводимость была использована схема полевого транзистора, представленная на рис. 4. Толщина запорного слоя оксида кремния составляла 100 нм. Как было сказано выше, при высоких напряжениях сток-исток и напряжениях смещения в пленках возможны электрические пробои, поэтому измерительные параметры выбирались из условия стабильной работы измерительной ячейки.

В пленках ОГ при частичном восстановлении можно наблюдать как протонную, так и отрицательно-ионную проводимости [29,30]. В данной работе в пленках ОГ исследовался полевой эффект лишь для протонной проводимости, поэтому пленки ОГ высушивались при комнатной температуре и восстановление не осуществлялось.

На рис. 5 приведены токовые характеристики протонной проводимости транзистора - в образце присутствует лишь не восстановленный ОГ, поэтому электронная проводимость отсутствует; на затвор $(G)$ подавалось ступенчатое напряжение от 0 до $\pm 12 \mathrm{~B}$ через $1.5 \mathrm{~B}$. $\mathrm{B}$ сухой атмосфере (RH 7\%) при напряжении исток-сток $U_{S D}=1$ В ток отсутствует $\left(i_{S D}<1 \mathrm{HA}\right)$ даже при подаче на затвор напряжения от -12 до +12 В. При помещении транзистора во влажную атмосферу в образце появляется ток, обусловленный протонной проводимостью.

На рис. 5, а представлен экспериментальный фрагмент измеряемого тока сток-исток $\left(i_{S D}\right)$ при подаче на затвор вначале отрицательного, а затем положительного напряжения смещения с шагом $1.5 \mathrm{~B}$. При отрицательном напряжении смещения ток существенно увеличивается, что подтверждает его протонный характер, а последующая подача на затвор (времена $t>100 \mathrm{c}$ ) положительного напряжения также возникает ток, который можно отнести к отрицательно-ионному току, обусловленному движением отрицательных ионов - например, ионов кислорода. В этот ток может давать вклад и электронно-дырочная составляющая. Процесс миграции ионов кислорода общепринят для ионных проводников и оксидов металлов [44]. Для пленок ОГ протонный ток на порядок больше, чем отрицательно-ионный (рис. 5), и этот эффект можно объяснить различной подвижностью носителей заряда - в первом случае это лег- 

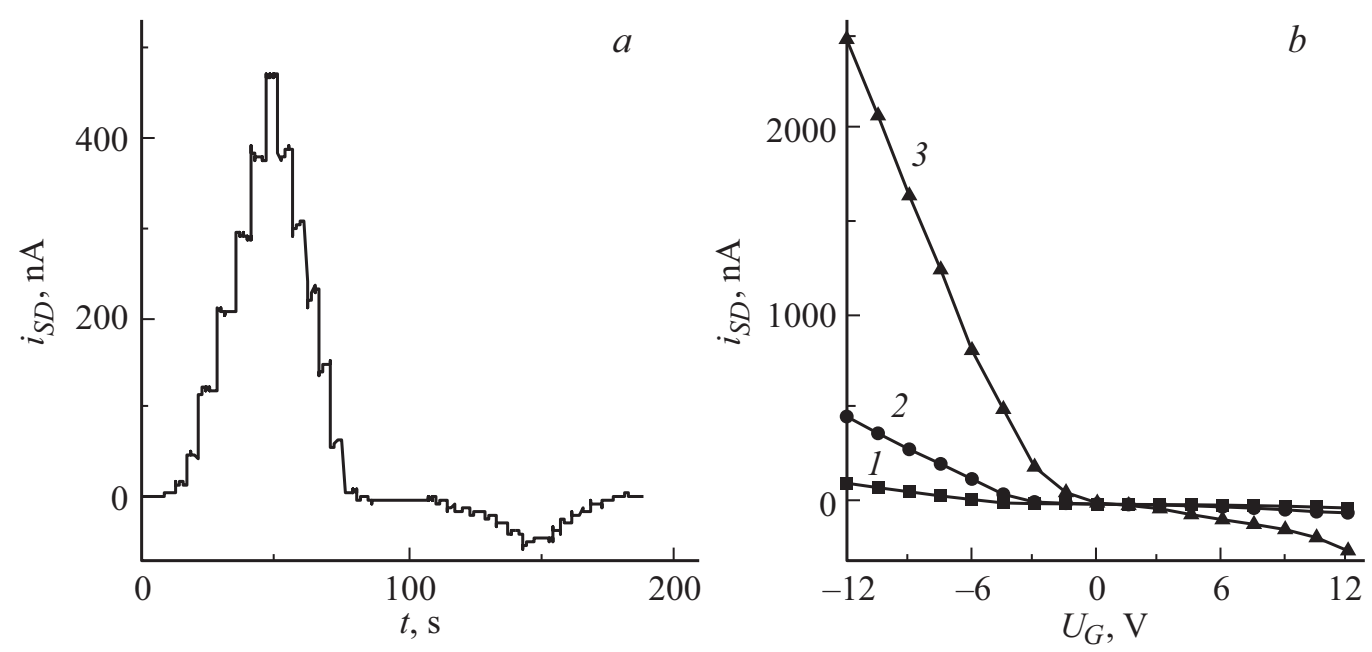

Рис. 5. $a$ - зависимость тока исток-сток $\left(i_{S D}\right)$ в пленке ОГ от ступенчатого во времени изменения напряжения на затворе $U_{G}$ : $0 \rightarrow(-12 \mathrm{~B}) \rightarrow 0 \rightarrow(+12 \mathrm{~B}) \rightarrow 0$, шаг $1.5 \mathrm{~B}\left(\mathrm{RH} 53 \%, U_{S D}=0.5 \mathrm{~B}\right) . b-$ токовые характеристики при изменении напряжения смещения и при различных влажностях RH: $1-35 \%, 2-53 \%, 3-75 \%$; $U_{S D}=0.5 \mathrm{~B}$.
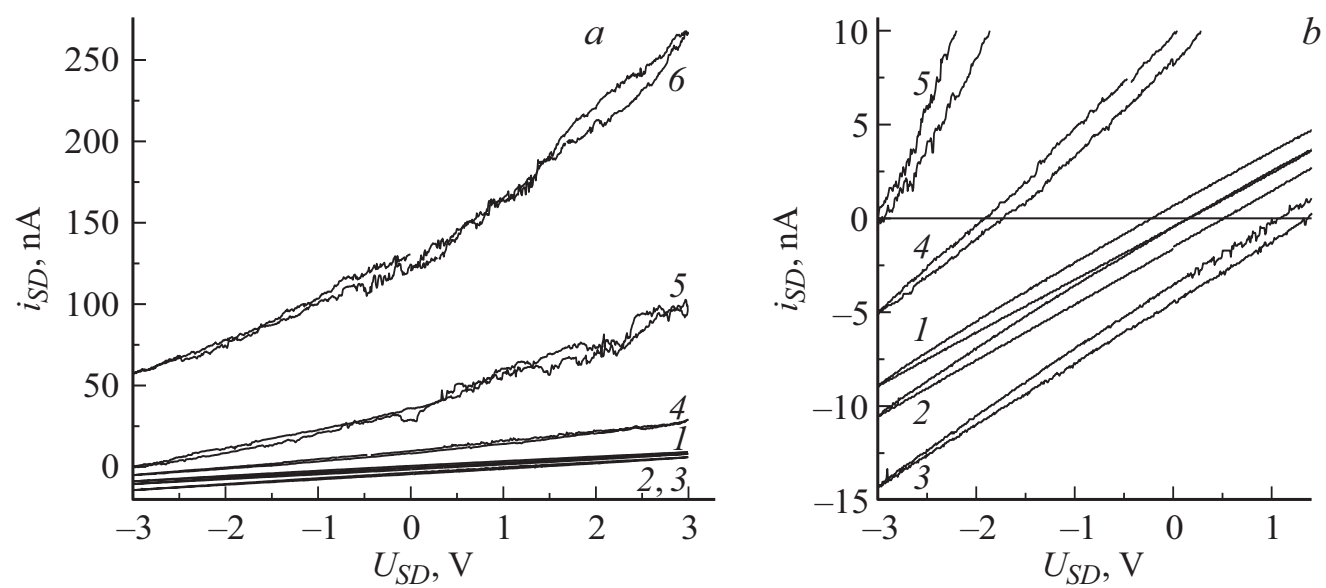

Рис. 6. $a-$ вольт-амперные характеристики протонной проводимости в пленке ОГ при циклическом линейном изменении напряжения $U_{S D}$ при напряжениях на затворе $U_{G}=0(1),+6(2),+12(3),-6(4),-9(5),-12$ В (6). $b-$ начальный участок характеристик. RH $35 \%$.

кие протоны, а во втором случае это отрицательно заряженные атомы (или молекулы) кислорода. Можно также предположить, что играет роль и энергия активации образования этих носителей заряда. На рис. 5, $b$ приведены токовые характеристики в зависимости от напряжения смещения при различной влажности, эти характеристики согласуются с зависимостью протонного тока от влажности (рис. $1, a)$.

На рис. 6 приведены вольт-амперные характеристики транзистора на основе ОГ при различных напряжениях смещения $\left(U_{G}\right)$. При положительных напряжениях смещения токовые характеристики практически линейны, а при больших отрицательных напряжениях смещения эти характеристики становятся нелинейными. Гистерезисных явлений при скорости изменения напряжения $\Delta U_{S D}=10 \mathrm{MB} / \mathrm{c}$ практически не наблюдается. Из начальных участков на рис. $6, b$ видна значительная величина отрицательно-ионного тока при положительном напряжении смещения (кривые 2 и 3 ).

В пленках нафиона полевой эффект исследовался при напряжении $U_{S D}=0.5 \mathrm{~B}$, а на затвор подавалось ступенчатое напряжение $U_{G}$ от 0 до $\pm 12 \mathrm{~B}$ через $1.5 \mathrm{~B}$, результаты измерений представлены на рис. 7.

Токовые характеристики в пленках нафиона аналогичны характеристикам в пленках ОГ, однако можно наблюдать и значительные количественные различия этих характеристик. Отрицательно-ионный ток в нафионовом варианте (рис. 7, кривая 3) на 2 порядка меньше протонного тока и значительно меньше отрицательно-ионного тока в пленках ОГ. Можно предположить, что в случае нафиона энергия образования (или глубина ловушки) отрицательного иона $\left(\mathrm{O}^{-}\right)$значительно больше, чем в пленке ОГ. Сравнивая результаты рис. 7 с результатами для ОГ (рис. 5), можно видеть, что при одинаковых 


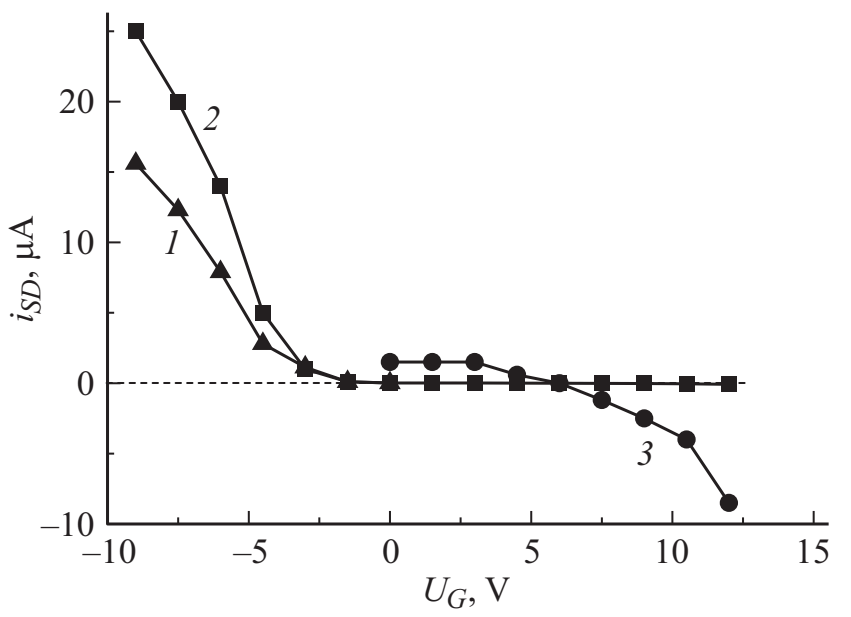

Рис. 7. Полевые зависимости тока $i_{S D}$ в пленке нафиона при различной влажности: $1-35 \%, 2-53 \%, 3-$ другой масштаб кривой $2\left(i_{S D} \times 100\right)$.

напряжениях смещения усиление протонного тока в случае нафиона на 1-2 порядка больше, чем для ОГ.

Эти выводы подтверждаются вольт-амперными характеристиками протонной проводимости в пленке нафиона, представленными на рис. 8. Как следует из рис. 8, вольт-амперные характеристики пленок нафиона подобны характеристикам пленок ОГ (рис. 6), однако значения токов при отрицательном смещении в случае нафиона значительно выше, чем в ОГ при тех же условиях. Это можно объяснить более высокой проводимостью пленок нафиона во влажной атмосфере по сравнению с пленками ОГ.

Наши данные согласуются с результатами других авторов по измерению эффекта поперечного электрического поля в материалах с протонной проводимостью: в полисахаридах протонная проводимость проявляется при влажности $\mathrm{RH}>50 \%$ [31], а в нафионе [32] на- блюдался полевой эффект, но влажность образца не оценивалась.

Мы оценили дрейфовую подвижность протонов в пленках ОГ и нафиона, исходя из общего соотношения для проводимости $\sigma=n e \mu$ и данных по температурной зависимости удельной электропроводности (рис. 3). При $20^{\circ} \mathrm{C}$ для ОГ $\sigma_{1}=10^{-5} \mathrm{C} / \mathrm{cm}$, для нафиона $\sigma_{2}=5 \cdot 10^{-4} \mathrm{C} / \mathrm{cm}$. Концентрацию протонов при наличии затворного смещения $U_{G}$ оценивали в соответствии с работой [15] и с учетом толщин пленок $d_{1}=1$ мкм, $d_{2}=2$ мкм получили концентрации $n_{1}=2.6 \cdot 10^{16} \mathrm{~cm}^{-3}$ и $n_{2}=8 \cdot 10^{15} \mathrm{~cm}^{-3}$ при затворных смещениях $U_{G 1}=-12 \mathrm{~B}$ и $U_{G 2}=-7.5 \mathrm{~B}$. Далее легко получить подвижности: $\mu_{1}=2.4 \cdot 10^{-3} \mathrm{~cm}^{2} / \mathrm{B} \cdot \mathrm{c}$ и $\mu_{2}=0.4 \mathrm{~cm}^{2} / \mathrm{B} \cdot \mathrm{c}$. Значение $\mu_{1}$ для пленок ОГ находится в качественном согласии с результатами работы по подвижности протонов во льду [45], тогда как для нафиона подвижность протонов на 2-3 порядка величины больше.

\section{4. Заключение}

Не восстановленные пленки ОГ во влажной атмосфере ведут себя как типичные протонные проводники, хотя и обладают значительно меньшей проводимостью. Используя протонную проводимость ОГ и нафиона, в схеме полевого транзистора можно значительно увеличить ток при подаче на затвор отрицательного смещения. Отличительной особенностью пленок ОГ от пленок нафиона в модели полевого транзистора является значительный ток, обусловленный отрицательноионной проводимостью при положительном поперечном электрическом поле. Следует обратить внимание, что использование ОГ в качестве изолятора в электронных устройствах может вносить значительные погрешности при изменении влажности окружающей среды за счет появления протонной проводимости.
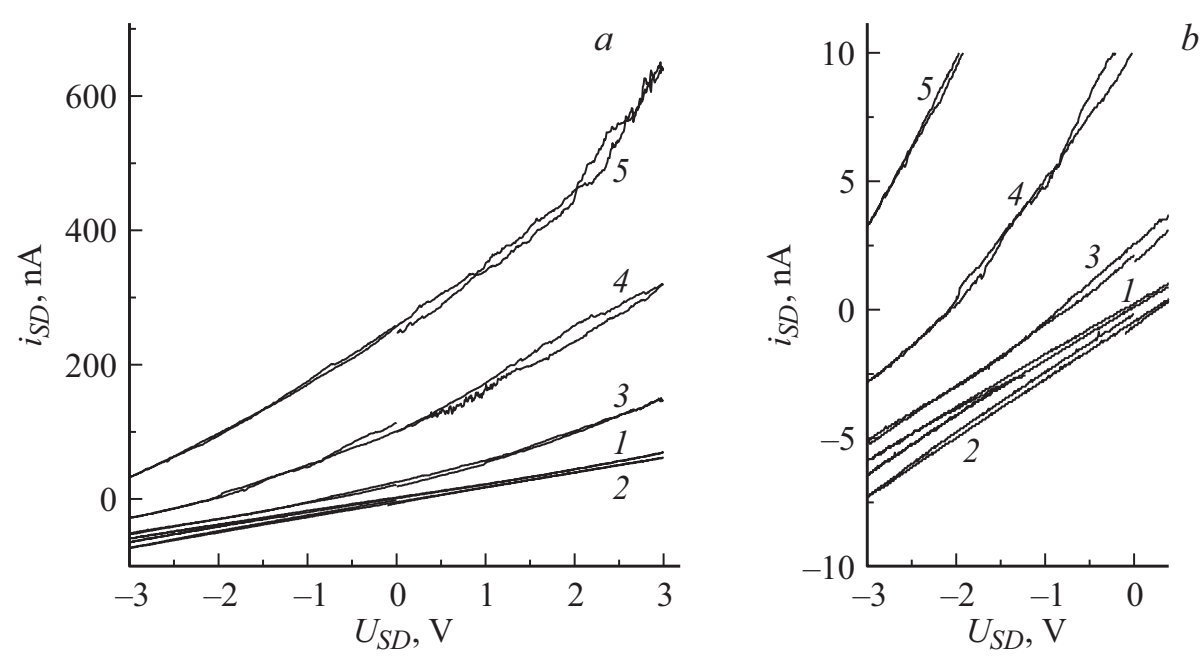

Рис. 8. $a-$ вольт-амперные характеристики протонной проводимости в пленке нафиона в зависимости от напряжения на затворе: $U_{G}=0(1),+12(2),-6(3),-9(4),-12$ B (5). $b$ - начальный участок характеристик $(a)$. RH $35 \%$. 


\section{Список литературы}

[1] O.C. Compton, S.B.T. Nguyen. Small, 6, 711 (2010).

[2] T. Ramanathan, A.A. Abdala, S. Stankovich, D.A. Dikin, M. Herrera-Alonso, R.D. Piner, D.H. Adamson, H.C. Schniepp, X. Chen, R.S. Ruoff, S.T. Nguyen, I.A. Aksay, R.K. Prud'Homme, L.C. Brinson. Nature Nanotechnol., 3, 327 (2008).

[3] F. Perrozzi, S. Prezioso, L. Ottaviano. J. Phys.: Condens. Matter, 27, 013002 (2015).

[4] D.A. Dikin, S. Stankovich, E.J. Zimney, R.D. Piner, G.H.B. Dommett, G. Evmenenko, S.T. Nguyen, R.S. Ruoff. Nature, 448, 457 (2007).

[5] X. Wu, M. Sprinkle, X. Li, F. Ming, C. Berger, W.A. de Heer. Phys. Rev. Lett., 101, 026801 (2008).

[6] G. Eda, Y.-Y. Lin, C. Mattevi, H. Yamaguchi, H.-A. Chen, I.S. Chen, C.-W. Chen, M. Chhowalla. Adv. Mater., 22, 505 (2010).

[7] L.W.K. Lee, Y.-Y. Sun, M. Lucking, Z. Chen, J.J. Zhao, S.B. Zhang. ACS Nano, 3, 2995 (2009).

[8] B.S. Paratala, B.D. Jacobson, S. Kanakia, L.D. Francis, B. Sitharaman. PLoS ONE, 7, e38185 (2012).

[9] И.В. Антонова, И.А. Котин, В.И. Попов, Ф.Д. Васильева, А.Н. Капитонов, С.А. Смагулова. ФТП, 50, 1086 (2016) [Simiconductors, 50, 1065 (2016)].

[10] W. Gao, N. Singh, L. Song, Z. Liu, A.L. Reddy, L. Ci, R. Vajtai, Q. Zhang, B. Wei, P.M. Ajayan. Nature Nanotechnol., 6, 496 (2011).

[11] Y.M. Shulga, S.A. Baskakov, V.A. Smirnov, N.Y. Shulga, K.G. Belay, G.L. Gutsev. J. Power Sources, 245, 33 (2014).

[12] V.A. Smirnov, N.N. Denisov, A.E. Ukshe, Yu.M. Shulga. Chem. Phys. Lett., 583, 155 (2013).

[13] V.A. Smirnov, N.N. Denisov, N.N. Dremova, Y.M. Vol'fkovich, A.Y. Rychagov, V.E. Sosenkin, K.G. Belay, G.L. Gutsev, N.Yu. Shulga, Yu.M. Shulga. Appl. Phys. A, 117, 1859 (2014).

[14] M.R. Karim, K. Hatakeyama, T. Matsui, H. Takehira, T. Taniguchi, M. Koinuma, Y. Matsumoto, T. Akutagawa, T. Nakamura, S.-I. Noro, T. Yamada, H. Kitagawa, S. Hayami. J. Am. Chem. Soc., 135, 8097 (2013).

[15] K.S. Novoselov, A.K. Geim, S.V. Morozov, D. Jiang, Y. Zhang, S.V. Dubonos, I.V. Grigorieva, A.A. Firsov. Science, 306, 666 (2004).

[16] M. Lemme. Sol. St. Phenomena, 156, 499 (2010).

[17] F. Schwierz. Nature Nanotechnol., 5, 486 (2010).

[18] A.H. Castro Neto, F. Guinea, N.M.R. Peres, R.S. Novoselov, A.K. Geim. Rev. Mod. Phys., 81, 109 (2009).

[19] J.-H. Chen, C. Jang, S. Xiao, M. Ishigami, M.S. Fuhrer. Nature Nanotechnol., 3, 206 (2008).

[20] I. Childres, L.A. Jauregui, M. Foxe, J. Tian, R. Jalilian, I. Jovanovic, Y.P. Chen. Appl. Phys. Lett., 97, 173109 (2010).

[21] И.В. Антонова, И.А. Котин, В.И. Попов, Ф.Д. Васильева, А.Н. Капитонов, С.А. Смагулова. ФТП, 50, 1086 (2016). [Semiconductors, 50, 1065 (2016)].

[22] G. Eda, A. Nathan, P. Wöbkenberg, F. Colleaux, T.D. Ghaffarzadeh, K. Anthopoulos, M. Chhowalla. Appl. Phys. Lett., 102, 133108 (2013).

[23] B. Standley, A. Mendez, E. Schmidgall, M. Bockrath. Nano Lett., 12, 1165 (2012).
[24] S.-K. Lee, H.Y. Jang, S. Jang, E. Choi, B.H. Hong, J. Lee, S. Park, J.-H. Ahn. Nano Lett., 12, 3472 (2012).

[25] A.N. Aleshin, I.P. Shcherbakov, A.S. Komolov, V.N. Petrov, I.N. Trapeznikova. Organic Electron., 16, 186 (2015).

[26] M.A. Mamo, A.O. Sustaita, N.J. Coville, I.A. Hümmelgen. Organic Electron., 14, 175 (2013).

[27] T.-W. Kim, N. Cernetic, Y. Gao, S. Bae, S. Lee, H. Ma, H. Chen, A.K.-Y. Jen. Organic Electron., 15, 2775 (2014).

[28] Y. Park, D. Gupta, C. Lee, Y. Hong. Organic Electron., 13, 2887(2012).

[29] В.А. Смирнов, А.Д. Мокрушин, В.П. Васильев, Н.Н. Денисов, К.Н. Денисова. Письма ЖТФ, 13, 18 (2016). [Techn. Phys. Lett., 42, 671 (2016)].

[30] V.A. Smirnov, A.D. Mokrushin, V.P. Vasiliev, N.N. Denisov, K.N. Denisova. Appl. Phys. A, 122, 513 (2016).

[31] C. Zhong, Y. Deng, A.F. Roudsari, A. Kapetanovic, M.P. Anantram, M. Rolandi. Nature Commun., 2, 476 (2011).

[32] K.L. Riskey. http://dspace.library.colostate.edu/webclient/ deliverymanager/digitool_items/csm01_storage/2013/06/15/ file_1/207313

[33] A.M. Deml, A.L. Bunge, M.A. Reznikov, A. Kolessov, R.P. O’Hayre. J. Appl. Phys., 111, 074511 (2012).

[34] M.A. Hickner, H. Ghassemi, Y.S. Kim, B.R. Einsla, J.E. McGrath. Chem. Rev., 104, 4587 (2004).

[35] K.A. Mauritz, R.B. Moore. Chem. Rev., 104, 4535 (2004).

[36] R.H. Alonso, L. Estevez, H. Lian, A. Kelarakis, E.P. Giannelis. Polymer, 50, 2402 (2009).

[37] S. Seesukphronrarak, A. Ohira. Chem. Commun., 45, 4744 (2009).

[38] S. Ochi, O. Kamishima, J. Mizusaki, J. Kawamura. Solid State Ionics, 180, 580 (2009).

[39] R. Kumar, M. Mamlouk, K. Scott. Int. J. Electrochem., Article ID 434186 (2011).

[40] S. Hu, M. Lozada-Hidalgo, F.C. Wang, A. Mishchenko, F. Schedin, R.R. Nair, E.W. Hill, D.W. Boukhvalov, M.I. Katsnelson, R.A.W. Dryfe, I.V. Grigorieva, H.A. Wu, A.K. Geim. Nature, 516, 227 (2014).

[41] L.M. Onishi, J.M. Prausnitz, J. Newman. J. Phys. Chem. B, 111, 10166 (2007).

[42] Y. Sone, P. Ekdunge, D. Simonsson. J. Electrochem. Soc., 143, 1254 (1996).

[43] S. Hink, N. Wagner, W.G. Besslerm, E. Roduner. Membranes, 2, 237 (2012).

[44] K.D. Kreuer. Annu. Rev. Mater. Res., 33, 333 (2003).

[45] M. Kunst, J.M. Warman. Nature, 288, 465 (2980).

Редактор Л.В. Шаронова 


\section{A field effect transistor with proton conductivity of graphene oxide and Nafion films}

V.A. Smirnov ${ }^{1}$, A.D. Mokrushin ${ }^{2}$, N.N. Denisov ${ }^{1}$, Yu.A. Dobrovolskii ${ }^{1}$

${ }^{1}$ Institute of Problems of Chemical Physics,

Russian Academy of Sciences,

142432 Chernogolovka, Moscow Region, Russia

${ }^{2}$ Institute of Microelectronics Technology

and High Purity Materials,

Russian Academy of Sciences,

142432 Chernogolovka, Moscow Region, Russia

Abstract Proton conduction in graphene oxide (GO) and Nafion films were investigated depending on the humidity and the voltage at the electrodes in the FET model. The electrical properties of the films are similar, but the mobility of positive charges in the Nafion and the current gain of 2-3 orders of magnitude higher than in GO. Negative-ion current in GO films with a positive voltage bias is appreciable by proton (up to $\sim 10 \%$ ), while in films of Nafion it practically absent $(<1 \%)$. 